\title{
Pulmonary artery and left atrial appendage anatomical relationship using electrocardiogram- -gated computed tomography: An important aspect of left atrial appendage occlusion
}

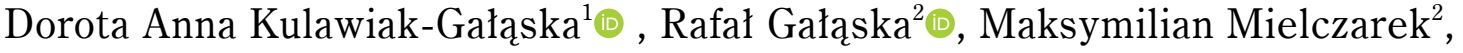 \\ Piotr Drewla ${ }^{2}$, Miłosz Jaguszewski ${ }^{2}$, Marcin Fijałkowski ${ }^{2}$, Michał Studniarek ${ }^{1}$ \\ ${ }^{1}$ Department of Radiology, Medical University of Gdansk, Poland \\ ${ }^{2} 1^{\text {st }}$ Department of Cardiology, Medical University of Gdansk, Poland
}

\section{This paper was guest edited by Prof. Wojciech Wojakowski}

Atrial fibrillation (AF) is an important cause of morbidity and mortality worldwide. The Framingham study and other studies have demonstrated a fivefold increase in overall stroke risk associated with AF [1]. Vitamin $\mathrm{K}$ antagonists have been shown to reduce stroke or systemic thromboembolism by $64 \%$ and all-cause mortality by $26 \%$. The newer non-vitamin $\mathrm{K}$ antagonist oral anticoagulants give an additional reduction effect with better adherence observed. Unfortunately, chronic anticoagulation is associated with a significant risk of major bleeding. Patients who have contraindications to anticoagulant treatment may derive benefit from left atrial appendage (LAA) occlusion. Transcatheter LAA closure has emerged as a potential alternative to oral anticoagulation in $\mathrm{AF}$ patients and contraindications for long-term oral anticoagulation. In recent years, it has been reported in the literature a few cases of pulmonary artery (PA) wall perforation leading to cardiac tamponade and even death following by an appendage closure using Amplatzer Cardiac Plug (ACP) or Amplatzer Amulet Occluder (AAO) [2,3]. One of those reports revealed a close anatomical relationship between LAA and PA as the most likely explanation for this complication, based on the preprocedural cardiac computed tomography (CT) examination [4]. The aim of the present study was to assess the anatomi- cal relationship and the distance between LAA and PA by means of electrocardiogram (ECG)-gated CT.

Data of consecutive 55 patients (mean age $63.1 \pm 10.7$; 30 females, 25 males), referred to coronary CT angiography were analysed. Only CT examinations comprising an entire cardiac cycle analysis were included in the study. All patients were in sinus rhythm after having fasted for 3 hours prior to the CT scan. The CT was performed during inspiration, scanning from the aortic arch to the diaphragm using a 128-row dual source CT scanner (Siemens Somatom Flash) with ECG gating. A retrospective ECG-triggered scan protocol with temporal resolution of $75 \mathrm{~ms}$ was used. For all patients, $80 \mathrm{~mL}$ of nonionic contrast medium was injected at rate of $6 \mathrm{~mL} / \mathrm{min}$ using a triphasic injection protocol. The region of interest was set in the ascendens aorta to ensure adequate opacification of the left cardiac chambers. Images were reconstructed with a slice thickness of $0.6 \mathrm{~mm}$ both during ventricular systole and diastole. Analysis was performed using axial source images and multi-planar reformats. The following measurements were taken: the minimal distance from the LAA to the PA (minD), the distance form LAA ostium to the location of the closest proximity of LAA and PA (depth_minD), distance from the LAA to the PA measured at $10 \mathrm{~mm}$ (D10) and $15 \mathrm{~mm}$ (D15) from the LAA

Address for correspondence: Dorota Kulawiak-Gałąska, MD, PhD, Department of Radiology, Medical University of Gdansk, ul. Smoluchowskiego 17, 80-214 Gdańsk, Poland, tel: +48 5834936 80, fax: +48 5834936 80, e-mail: dorkul@gumed.edu.pl

Received: 7.05.2020

Accepted: 19.01.2021

This article is available in open access under Creative Common Attribution-Non-Commercial-No Derivatives 4.0 International (CC BY-NC-ND 4.0) license, allowing to download articles and share them with others as long as they credit the authors and the publisher, but without permission to change them in any way or use them commercially. 


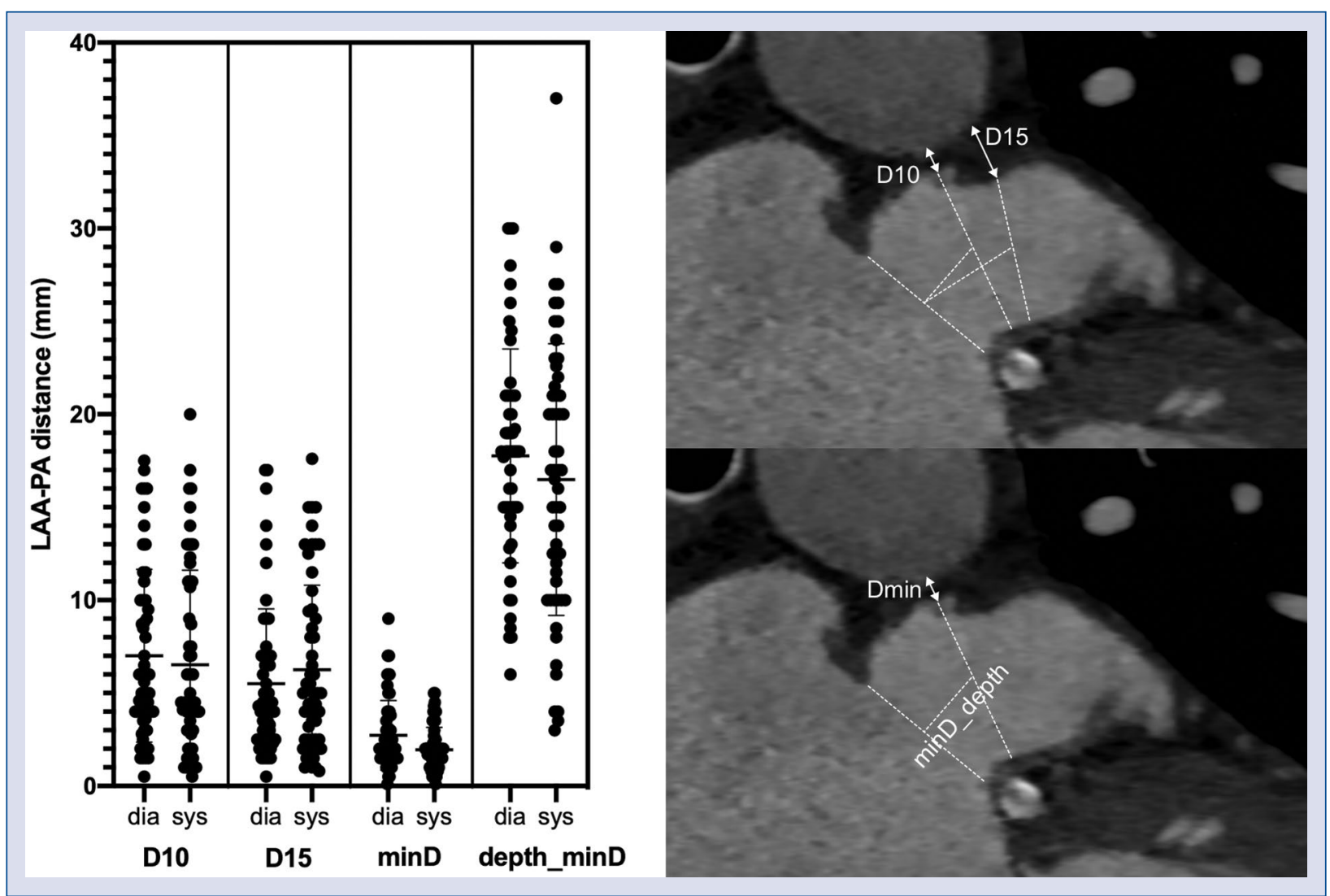

Figure 1. The distance from left atrial appendage (LAA) to the pulmonary artery (PA); D10 - the distance from the LAA to the PA measured at $10 \mathrm{~mm}$ from the LAA ostium; D15 - the distance from the LAA to the PA measured at $15 \mathrm{~mm}$ from the LAA ostium; minD — the minimal distance from the LAA to the PA; depth-minD — the distance form LAA ostium to the location of the closest proximity of LAA; dia - ventricular diastole; sys — ventricular systole.

ostium. The distance $10 \mathrm{~mm}$ and $15 \mathrm{~mm}$ were chosen as markers of a potential landing zone for LAA occluder devices. All measurements were averaged and taken by an experienced in cardiac CT radiologist and cardiologist blinded to patient clinical data. They were analysed separately during ventricular diastole and systole.

The results are presented in the Figure 1 as a scatter plot with marked mean value and standard deviation. Direct contact of proximal LAA and PA defined as the distance between their lumens $<1 \mathrm{~mm}$ was observed in $8(14.5 \%)$ patients and varied from $2(3.6 \%)$ in ventricular diastole up to $6(10.9 \%)$ in ventricular systole in a potential landing zone.

There are several case reports describing PA perforation following LAA closure, and majority of them concerned the older ACP device. In those situations, there were suggestions that small anchors used for device stabilization in some situations may have had a higher risk of extending through the LAA wall and damaging the PA wall. In the newer AAO, some parts of the plug were redesigned including stabilizing anchors [5]. What is interesting, time from plug implantation to pulmonary perforation varied from 3 hours [6] till 6 months after the procedure [7], what brings potential risk to the patient of having cardiac tamponade out of the hospital. The current analysis of cardiac anatomy based on CT examinations, confirmed close proximity of the pulmonary trunk and potential landing zone for occluder devices in LAA in a significant number of patients. Direct contact of proximal LAA and PA defined as the distance between their lumens $<1 \mathrm{~mm}$ was observed in $8(14.5 \%)$ patients. It was significantly lower when compared with results of Halkin et al. [6]. Halkin et al. [6] found direct contact between LAA and $\mathrm{PA}$ in $28 \%$ of patients qualified to pulmonary vein isolation and with AF. The analysis was performed during ventricular diastole [6]. In present study patients were in sinus rhythm, but patients with sinus rhythm with a history of paroxysmal AF 
represent a significant number of patients referred to LAA occlusion. Good contractility of the LAA as the result of underlying sinus rhythm may play an important role in facilitating PA perforation in those patients [8]. Previous research has shown that LAA size significantly depends on loading conditions of the LA [9]. Therefore, the present results could be underestimated when compared with real-life conditions, due to the study group fasting for 3 hours prior to the CT scan. Analysis herein, underlines the importance of careful assessment of the anatomical relationships between LAA and surroundings structures before implantation of the device. Avoiding excessive oversizing seems to be an important part of the preprocedural planning in patients with direct contact between the atrial appendage and the PA. In selected patients the choice of a different closure device with smaller anchors may be the proper option [10]. Cardiac CT is an optimal method to achieve the abovementioned goals.

In conclusion, close proximity between the PA and a proximal part of the LAA occurs in a significant percentage of patients. Cardiac CT can be used to optimize risk assessment, procedure planning and device selection performed before LAA occlusion in relation to the probability of a potential PA injury.

\section{Conflict of interest: None declared}

\section{References}

1. Wolf PA, Abbott RD, Kannel WB. Atrial fibrillation as an independent risk factor for stroke: the Framingham Study. Stroke. 1991; 22(8): 983-988, doi: 10.1161/01.str.22.8.983, indexed in Pubmed: 1866765.

2. Zwirner J, Bayer R, Hädrich C, et al. Pulmonary artery perforation and coronary air embolism-two fatal outcomes in percuta- neous left atrial appendage occlusion. Int J Legal Med. 2017; 131(1): 191-197, doi: 10.1007/s00414-016-1486-1, indexed in Pubmed: 27815629.

3. Suwalski G, Wojnowski A, Mizerski J, et al. Delayed pulmonary artery perforation with left atrial appendage occluder hooks. Ann Thorac Surg. 2016; 101(2): e37-e39, doi: 10.1016/j.athoracsur.2015.09.026, indexed in Pubmed: 26777968.

4. Hanazawa K, Brunelli M, Saenger J, et al. Close proximity between pulmonary artery and left atrial appendage leading to perforation of the artery, tamponade and death after appendage closure using cardiac plug device. Int J Cardiol. 2014; 175(2): e35-e36, doi: 10.1016/j.ijcard.2014.04.260, indexed in Pubmed: 24838059 .

5. Grygier M, Wojakowski W, Smolka G, et al. [Left atrial appendage occlusion: consensus document of Association of Cardiovascular Interventions and Heart Rhythm Section of Polish Cardiac Society]. Kardiol Pol. 2018; 76(3): 677-697, doi: 10.5603/ KP.a2018.0057, indexed in Pubmed: 29441512.

6. Halkin A, Cohen C, Rosso R, et al. Left atrial appendage and pulmonary artery anatomic relationship by cardiac-gated computed tomography: Implications for late pulmonary artery perforation by left atrial appendage closure devices. Heart Rhythm. 2016; 13(10): 2064-2069, doi: 10.1016/j.hrthm.2016.07.015, indexed in Pubmed: 27430898.

7. Wang E, Lin WW, Xu XF, et al. Delayed presentation of pulmonary artery perforation by an Amulet left atrial appendage closure device. BMJ Case Rep. 2018; 2018, doi: 10.1136/bcr2018-227098, indexed in Pubmed: 30391926.

8. Chun KR, Bordignon S, Urban V, et al. Left atrial appendage closure followed by 6 weeks of antithrombotic therapy: a prospective single-center experience. Heart Rhythm. 2013; 10(12): 1792-1799, doi: 10.1016/j.hrthm.2013.08.025, indexed in Pubmed: 23973952 .

9. Korsholm K, Berti S, Iriart X, et al. Expert recommendations on cardiac computed tomography for planning transcatheter left atrial appendage occlusion. JACC Cardiovasc Interv. 2020; 13(3): 277-292, doi: 10.1016/j.jcin.2019.08.054, indexed in Pubmed: 31678086 .

10. Asmarats L, Rodés-Cabau J. A look at current and emerging nonpharmacologic stroke prevention therapies. Spectrum Devices Percutaneous Left Atrial Appendage Occlusion. 2018; 12(3): 34-39. 